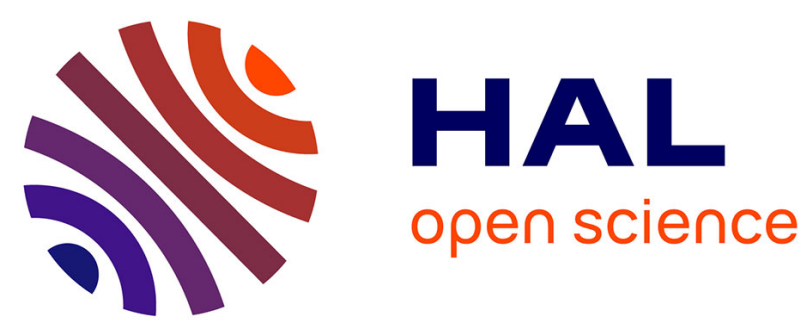

\title{
Le possible rôle de la néo-tectonique dans les variations relatives du niveau marin sur la presqu'île de Taman (Mer Noire, Mer d'Azov, Russie)
}

Eric Fouache, Alexeï V. Porotov, Christel Müller, Youri Gorlov

\section{- To cite this version:}

Eric Fouache, Alexeї V. Porotov, Christel Müller, Youri Gorlov. Le possible rôle de la néo-tectonique dans les variations relatives du niveau marin sur la presqu'île de Taman (Mer Noire, Mer d'Azov, Russie). Topoi Orient - Occident, 2001, 11 (2), pp.599 - 611. 10.3406/topoi.2001.1954 hal-01667305

\section{HAL Id: hal-01667305 \\ https://hal.parisnanterre.fr/hal-01667305}

Submitted on 19 Dec 2017

HAL is a multi-disciplinary open access archive for the deposit and dissemination of scientific research documents, whether they are published or not. The documents may come from teaching and research institutions in France or abroad, or from public or private research centers.
L'archive ouverte pluridisciplinaire HAL, est destinée au dépôt et à la diffusion de documents scientifiques de niveau recherche, publiés ou non, émanant des établissements d'enseignement et de recherche français ou étrangers, des laboratoires publics ou privés. 


\section{LE POSSIBLE RÔLE DE LA NEO-TECTONIQUE DANS LES VARIATIONS RELATIVES DU NIVEAU MARIN SUR LA PRESQU'ÎLE DE TAMAN * \\ (Mer Noire, Mer d'Azov, Russie)}

\section{Introduction}

La péninsule de Taman se rattache géologiquement à la terminaison occidentale du Caucase (Atlas géologique de la région de Krasnodar 1995). Cette péninsule est délimitée à l'Ouest par le détroit de Kertch, appelé Bosphore Cimmérien dans l'Antiquité, au nord par la mer d'Azov, le lac Méotide des anciens, et au sud par la Mer Noire, le Pont Euxin (fig. J). Des colonies grecques se sont implantées dans la région dès avant le $V^{e}$ siècle av. J.-C., comme en témoignent les vestiges archéologiques des cités d'Hermonassa, de Phanagoria, de Kepoi et Patrasys, pour ne citer que les plus connues. Une grande partie de ces vestiges sont aujourd'hui submergés, ce qui nous a conduit, dans le cadre de la mission franco-russe de Taman dirigée par Christel Müller et Youri Gorlov, à tenter de reconstituer les variations relatives du niveau marin depuis 6000 ans. Notre étude s'intéresse donc à la période qui suit l'inondation rapide de la Mer Noire. Celleci constituait jusqu'à cette époque une aire lacustre. L'arrivée des eaux marines par le détroit du Bosphore s'est déclenchée il y a 7500 ans, à partir du moment où le lac est connecté, via la Mer de Marmara, à la Méditerranée (RyAN - PITMAN 1999). Méthodologiquement nous avons procédé en 1998 et 1999 , grâce à un carottier hydraulique lourd, à des carottages en différents points de la péninsule, notamment dans le delta du Kouban et sur des cordons littoraux sableux. Sur la

* Remerciements. Cette étude, réalisée dans le cadre de la mission archéologique franco-russe de Taman, a bénéficié du financement du Ministère des affaires étrangères, de l'École Française d'Athènes, du CNRS, et de l'Académie des Sciences de Russie. 
base des observations sédimentologiques et des résultats de datations au Carbone 14, réalisées sur des coquilles marines, nos observations nous conduisent à reconstituer des variations relatives du niveau marin dont l'ampleur et la dissymétrie d'un point à l'autre de la péninsule ne peuvent s'expliquer que par l'effet de la néo-tectonique.

\section{Méthodologie}

Dans le cadre de notre étude, nous avons pris en compte des indicateurs sédimentologiques et malacologiques associés à des datations au Carbone 14 réalisées sur des coquilles marines. L'impossibilité d'exporter nos carottes, les restrictions techniques imposées par les services géologiques russes, les difficultés matérielles sur place ont rendu impossible une étude paléo-environnementale systématique des carottes en laboratoire. Pour ces raisons, nous avons privilégié l'observation in situ de la stratigraphie et nous avons procédé sur place à l'échantillonnage. Il en découle que nos reconstitutions des variations relatives du niveau marin souffrent d'une imprécision de l'ordre de $0,5 \mathrm{~m}$ à $1 \mathrm{~m}$. Néanmoins, l'ampleur des variations observées sur la verticale rend nos observations intéressantes.

Nous avons réalisé des carottages sur le cordon de Bugaz (S2), d'Anapa (S13) et de Tchouchtchka (S8) et dans le delta du Kouban (S1).

Les marqueurs archéologiques sont d'un faible intérêt dans le contexte de la péninsule de Taman dans la mesure où l'inventaire des vestiges archéologiques portuaires, indiscutablement en relation avec le niveau de la mer à une époque donnée, n'a jamais été réalisé. C'est la raison pour laquelle les méthodologies proposées - N.C. Flemming $(1969,1979)$ ou P.A. Pirazzoli (1976) -, utilisées par Éric Fouache en Turquie (FouACHE et al 1999), sont inutilisables ici.

Sur les cordons littoraux sableux nous avons pu identifier des niveaux sédimentaires caractéristiques de différents milieux qui, superposés, permettent de reconstituer plusieurs générations de niveaux marins. Il s'agit soit de sables grossiers de plage sur lesquelles se sont accumulées d'importantes thanatocénoses, soit de sédiments limoneux ou argilo-sableux caractéristiques de milieux lagunaires d'arrière cordons. Nous avons également réalisé des carottages à l'intérieur de la péninsule afin d'identifier la ligne de rivage correspondant au maximum de la transgression flandrienne.

L'extraction des coquilles et leur détermination ont été effectuées par l'équipe du professeur Kaplin, ainsi que les interprétations paléo-environnementales. (KAPLIN et al. 2001). Les datations au Carbone 14 ont été réalisées à l'Institut géologique de l'Académie des Sciences de Moscou et à la Faculté de 
Géographie de l'Université d'État de Moscou. La calibration a été menée selon le protocole élaboré par M. Stuiver et T.F. Brazianas (1993).

\section{Le contexte géologique}

La péninsule de Taman (fig. 1) est constituée de roches miocènes et pliocènes, essentiellement des argiles et des sables et, marginalement, on observe des affleurements de craie, notamment à la base du Cap Touzla et du cap Achilleion. L'orogenèse alpine, par sa tectonique compressive, est à l'origine de l'émersion de ces roches et de leur plissement selon des axes anticlinaux et synclinaux orientés Est-Sud-Est/Ouest-Nord-Ouest et du jeu de failles Nord/Sud. Le relief actuel de la presqu'île de Taman résulte de l'évolution à l'air libre, depuis la fin du Pliocène, des chaînons plissés et de l'action de la morphogenèse littorale et fluviale au cours du Pléistocène et surtout de l'Holocène. Les chaînons plissés ont été aplanis, tandis que, sur les axes anticlinaux, des phénomènes de diapirisme engendraient au Pléistocène des alignements de volcans de boue (ChNroukov 1986), à l'origine de cônes éruptifs dont les plus grands dépassent cent mètres de haut et occupent les points culminants de la topographie. Une partie de ces volcans de boue sont toujours en activité, ce qui témoigne de l'activité tectonique de la région.

\section{Résultats}

Le sondage S1 (fig. 2) indique que le maximum de la remontée postglaciaire se produit postérieurement à $5940 \pm 50 \mathrm{BP}$, datation au radiocarbone obtenue sur de la tourbe continentale à la base du sondage et surmontée de $6,5 \mathrm{~m}$ de dépôts marins, eux-mêmes recouverts de $5 \mathrm{~m}$ d'alluvions. À cette date, la péninsule de Taman forme une île.

Les sondages réalisés respectivement sur les cordons sableux de Bugaz (S2), d'Anapa (S12 et S13) et de Tchouchtchka (S8) (fig. 3), montrent une alternance de sédiments sableux bio-détritiques caractéristiques de plages et de sédiments à dominante argileuse riches en coquilles. Ce sont ces coquilles qui ont servi à réaliser des datations au Carbone 14 (Chart I). Les coquilles prélevées (Ostrea edulis, Donax trunculus, Chione gallina, Cerastoderma glaucum, Mytilus galloprovincialis, Scrobicularia plana, Chlamys glabra, mytilaster lineatus) appartiennent au milieu infra-littoral lagunaire et marin. Les datations, données ici en âge calendaire, montrent que la base des cordons sommitaux remonte au Moyen Âge : 1342 AD en S2 (-3,2 à -3,5 m), 1184 AD en $\mathrm{S} 13(-2$ à $-2,3 \mathrm{~m}), 1441$ en $\mathrm{S} 8(-2,8 \mathrm{~m})$. Elles indiquent, pour les autres cordons, une dissymétrie importante entre des marqueurs de niveaux marins contemporains. Il n'est que de comparer les fourchettes d'altitude d'un sondage à 
l'autre pour se rendre compte que ces différences atteignent plusieurs mètres (Tableau I).

\begin{tabular}{lll}
\multicolumn{1}{c}{$\mathrm{S} 8$} & \multicolumn{1}{c}{$\mathrm{S} 2$} & \multicolumn{1}{c}{$\mathrm{S} 13$} \\
$1441 \mathrm{AD}(-2,8 \mathrm{~m})$ & $1342 \mathrm{AD}(-3,2$ à $-3,5 \mathrm{~m})$, & $1184 \mathrm{AD}(-2$ à $-2,3 \mathrm{~m})$ \\
$2501 \mathrm{BC}(-3,2 \mathrm{~m})$ & $1310 \mathrm{AD}(-3,6-$ à $3,8 \mathrm{~m})$ & $827 \mathrm{BC}(-3,5$ à $-4 \mathrm{~m})$ \\
$3492 \mathrm{BC}(-4 \mathrm{à}-4,5 \mathrm{~m})$ & $649 \mathrm{AD}(-7$ à $-7,2 \mathrm{~m})$ & $1287 \mathrm{BC}(-5,5$ à $-6 \mathrm{~m})$ \\
$5959 \mathrm{BC}(-9$ à $-9,3 \mathrm{~m})$ & $200 \mathrm{BC}(-8,1$ à $-8,2 \mathrm{~m})$ & $1873 \mathrm{BC}(6,2$ à $6,7 \mathrm{~m})$ \\
& $740 \mathrm{BC}(-9$ à $-9,7 \mathrm{~m})$ & $2881 \mathrm{BC}(-7$ à $-7,5 \mathrm{~m})$
\end{tabular}

Tableau 1 - Répartition altitudinale des datations au Carbone 14 réalisées dans les sondages S8, S2 et S13

On distingue deux types de stratigraphie (fig. 3):

- Le premier montre la présence d'un cordon sableux édifié à partir de $4220 \pm 100$, s'exhaussant et progradant en parallèle avec un relèvement du niveau marin qui apparaît lent et continu, si l'on excepte une accélération autour de $1000 \mathrm{BC}$, jusqu'au niveau actuel. C'est la situation telle que nous la reconstituons sur le cordon sableux d'Anapa et en S13.

- Le second montre des alternances de dépôts sableux coquilliers caractéristiques du cordon de plage et de dépôts argileux liés à un milieu d'arrière cordon. Cela traduit des déplacements horizontaux et verticaux importants des cordons en S2 et S8.

Globalement, on constate une dissymétrie dans les cotes d'altitude de niveaux marins contemporains (Tableau I), qui ne peut être que d'origine tectonique. Cette dissymétrie révèle en outre une néotectonique différentielle. Le taux de subsidence actuel, estimé pour la région de Temrjuk entre $1-1,5 \mathrm{~mm} / \mathrm{an}$ (SHNuKov 1981), ne peut être étendu à l'ensemble de la péninsule. C'est la raison pour laquelle nous proposons des courbes de la variation du niveau marin relatif non corrigées. Nous comparons maintenant nos résultats avec ceux obtenus dans le delta du Kouban, sur la Mer d'Azov, et plus globalement sur le littoral de la Mer Noire.

\section{Discussion}

Une certitude émerge tout d'abord de nos résultats : le niveau marin actuel est le plus haut niveau jamais observé à l'Holocène sur la presqu'île de Taman. Nulle part on n'observe de cordons fossiles supérieurs au niveau de l'actuel et deux carottages, S6 et S7 (fig. 4), réalisés entre les cités de Kepoï et Tyrambe, là où Paromov (1993) envisageait un bras de mer dans l'Antiquité, montrent 
jusqu'à trois mètres sous le niveau actuel de la mer des colluvions d'origine continentale. Le mouvement apparent, tel qu'il apparaît dans les carottages S2, S8 et S13, si l'on fait abstraction de la subsidence, semble plutôt être celui d'une remontée continue du niveau marin réel.

La subsidence tectonique, attestée sur la péninsule, accentue localement l'ampleur de la variation verticale du niveau marin. La comparaison entre les profils sédimentaires observés en S13, S2 et S8 montre clairement que cette subsidence est plus forte au centre de la péninsule, c'est-à-dire la région des limans de Kiziltasch et d'Artanizovski. Le décalage atteint, aux alentours de 740 $\mathrm{BC}$, près de $8 \mathrm{~m}$ entre les niveaux marins contemporains observés entre $\mathrm{S} 2$ et $\mathrm{S} 8$ et $6 \mathrm{~m}$ entre $\mathrm{S} 2$ et $\mathrm{S} 13$. Ce qui établit un taux de subsidence sur 2700 ans de $2,6 \mathrm{~mm} / \mathrm{an}$ du cordon de Bugaz en S2, par rapport à la flèche de Tchouchtchka en $\mathrm{S} 8$. C'est bien la preuve d'une activité tectonique holocène dans une région où, jusqu'à présent, n'était prouvée qu'une activité tectonique pléistocène sur l'escarpement de faille au nord de la péninsule de Taman (NIKONOv 1994).

Quelle est la part réelle de l'eustatisme dans la remontée du niveau marin à partir de $3500 \mathrm{BC}$ ? On constate en effet que les cordons littoraux localisés dans la zone du pré-Kouban sur la mer d'Azov (IsMajlov et al. 1989), qui s'échelonnent de $5560 \pm 60 \mathrm{BP}(3610 \mathrm{BC})$ et $690 \pm \mathrm{BP}(1260 \mathrm{AD})$ sont tous dans une fourchette altitudinale identique située entre -2 et $+2 \mathrm{~m}$ autour du niveau moyen actuel de la mer d'Azov (FOUACHE et al. 2000). Le fait que les déplacements sur la verticale de la ligne de rivage ne correspondent pas entre la zone du préKouban, le cordon de Tchouchtchka et la région des limans de Kiziltasch et d'Artanizovski, confirme le rôle majeur de la tectonique dans ces variations. E.N. Nevessky (1970), supposait déjà le rôle de cette tectonique, déduite de l'étude de dépôts coquilliers prélevés par carottages sur les littoraux du Golfe de Karkinit, du golfe Kalamite et dans la région d'Anapa.

Nos résultats confirment ceux de Nevessky et remettent en cause la validité de la courbe des fluctuations du niveau marin, établie par K.K. Shilik (1997) (fig. 5, A), essentiellement à partir de marqueurs archéologiques et de quelques marqueurs géomorphologiques, et appliquée à la péninsule de Taman, comme à tout le littoral de la Mer Noire. À la suite de Fedorov (1971, 1981), K.K. Shilik (1997) considère qu'après une période durant laquelle le niveau marin s'est stabilisé à un niveau proche de l'actuel entre 6000 et 4000BP, une régression marine de l'ordre de $5 \mathrm{~m}$ à $6 \mathrm{~m}$ appelée « régression phanagorienne » s'est produite, suivie durant le premier millénaire ap. J.-C. d'une nouvelle transgression qui dépasserait le niveau actuel de $0,5 \mathrm{~m}$ à $1 \mathrm{~m}$. Cette régression Phanagorienne est acceptée par de nombreux auteurs sauf Nevessky (PIRAzZOLi 1991) (fig. 5, C et D).

Le principal défaut de la reconstitution de K.K. Shilik, comme des autres tenants d'une primauté des mouvements eustatiques en Mer Noire, est de nier d'emblée l'influence de la tectonique sur les variations du niveau marin pour les 
3500 dernières années. Cette influence est estimée à moins d'un mètre (SHILIK 1997, p. 115). Il s'avère notamment que l'on n'a pas accordé assez d'importance au contexte tectonique des régions dans lesquelles on recherchait des marqueurs des niveaux marins. On constate par exemple que les marqueurs retenus pour définir la « régression phanagorienne », qu'il s'agisse de marqueurs archéologiques ou de marqueurs géomorphologiques, sont tous situés dans des zones deltaïques affectées par de la subsidence (delta du Dnierp, delta du Danube par exemple). À l'inverse, les principaux marqueurs retenus pour caractériser le pic de transgression marine, présumée se produire postérieurement à la « régression phanagorienne », sont tous localisés sur la péninsule de Kertch, dont A. Nikonov (1994) a montré qu'elle était en surrection au quaternaire. De même, la valeur de 9 mètres en dessous du niveau actuel de la mer donnée comme maximum de la régression phanagorienne correspond à ce que nous mesurons en $\mathrm{S} 2$ dans une zone de subsidence maximale dans la péninsule de Taman.

La méthode qui consiste à croiser marqueurs archéologiques et marqueurs géomorphologiques pour établir à une date précise la position relative du niveau marin est une bonne méthode. Encore convient-il de retenir des marqueurs archéologiques in situ, incontestablement en relation avec le niveau de la mer au moment de leur construction. Il est d'ores et déjà incontestable que le facteur néo-tectonique a été négligé.

Il est donc nécessaire de réaliser de nouveaux inventaires régionaux des marqueurs des niveaux marins en Mer Noire, en prenant soigneusement en compte le contexte géologique et géodynamique. C'est seulement alors que l'on pourra proposer une reconstitution viable des fluctuations du niveau marin à l'Holocène moyen et récent.

\section{Conclusion}

Les carottages réalisés sur les cordons littoraux de la péninsule de Taman et dans les deltas du Kouban démontrent l'importance de la néo-tectonique holocène dans les variations verticales de la ligne de rivage dans cette région. Nous sommes amenés, en comparant nos résultats avec ceux établis par K.K. Shilik (1993), à remettre en question la réalité des phases de transgression et de régression sensées rythmer les variations du niveau moyen de la Mer Noire depuis l'Holocène moyen et à constater qu'incontestablement le facteur néotectonique a été jusqu'à présent largement sous-estimé. 


\section{Bibliographie}

Atlas Géologique de la région de Krasnodar, 1995.48p (en russe)

BALABANOV et al. 1981

I.P. BALABANOV, B.D. KVIRKELIYA, A.B. OSTROVSKY, Noveishaya istoriya formirovaniya inzhenerno-geologicheskikh uslovii $i$ dolgosrochnyi prognoz razvitiya beregovoi zony poluostrova Pitsunda, Tbilisi Mitsnierba.

FLEMMING 1969

N.C. Flemming, «Archaeological evidence for eustatic change of sea level and earth movements in the Western Mediterranean in the last 2000 years », Geological Society of America, Special Paper 109, p. 1-125.

FLEMMING 1979

N.C. FLEMMING, «Archaeological indicators of sea level », in: Les indicateurs de niveaux marins, Oceanis 5, Fasc. h/série, p. 149-166.

FOUACHE et al. 1998

É. FOUACHE, C. MÜLlER, Y. GORLOV, « Approche Géo-archéologique des paysages de la presqu'île de Taman (Mer Noire. Russie) », in : Cité et Territoires II, Environnement antique et Aménagement, Statut et Territoire des cités antiques, Ville de Béziers, ISTA, Service Régional de l'Archéologie, CNRS, 24-25 octobre 1997, Actes du Colloque, p. 141-153.

FOUACHE et al. 1999

É. Fouache, P. Sibella, R. DAlongeville, «Holocene variations of the shoreline between Antalya and Andriake (Turkey) », International Journal of Nautical Archaeology 28/4, p. 305-318.

FOUACHE et al. 2000

É. FouAChe, C. MÜller, Y. Gorlov, V. Gaibov, A. POROTOV, «Geoarchaeological study of theTaman peninsula and the Kouban delta (Black Sea, Azov Sea, Russia) », in: Geoarchaeology of the landscapes of classical antiquity. International symposium Gent, 23-24 Octobre 1998. Université de Gent, Working Group on Geoarchaeology of the IAG, Actes du Colloque, p. 97-104.

ISMAJLOV et al. 1989

J. ISMAJlov, K. ARSLANOV, T. TERTYCNAJA, S. CERnOV, « Restitutions et datations des lignes de rivage dans le delta du Kouban à l'Holocène ", Journal de l'Université de Léningrad, série VII, 3 (21), p. 61-69 (en russe).

\section{NEVESSKY 1970}

E.N. NEVESSKY, « Holocene history of the coastal shelf zone of the USSR in relation with processes of sedimentation and condition of concentration of useful minerals », Quaternaria 12, p. 78-86.

NIKONOV 1994

A. NIKONOV, «Les signes d'une activité tectonique récente dans les zones de faille de la mer d'Azov méridionale et du détroit de Kertch », Geoteknonika 5, p. 16-27 (en russe).

OSTROVSKY et al. 1977

A.B. OSTROVSKy, S.A. IZMAYlov, I.P. Abalabanov, S.I. SKIBA, N.G. SKRYABINA, S.A. ARSLANOV, N.A. GEY et N.I. SUPRUNOVAUPRUNOVa, « New data on the paleohydrological regime of the Black Sea in the Upper Pleistocene and Holocene », in : Paleogeography and deposits of the pleistocene of the Southern Sea of the USSR, Moscow, p. 131-141.

PAROMOV 1993

I. PAROMOV, «Principes de la mise en lumière de l'évolution du système d'occupation d'après l'exemple de la Péninsule de Taman », KSIA 210, p. 25-34. 
PIRAZZOLI 1976

P.A. PIRAZZOLI, «Les variations du niveau marin depuis 2000 ans », Mémoires $d u$ Laboratoire de Géomorphologie de l'ÉPHÉ 30, p. 1-421.

PIRAZZOLI 1991

P.A. PIRAzzolı, World Atlas of Holocene Sea-Level Changes. Elsevier Oceano graphy series, p. 58-300.

RYAN et al. 1999

W. RYAN, W. PITMAN, Noah's Flood: The new scientific discoveries about the event that changed history.

SHILIK 1997

K.K. SHILIK, «Oscillations of the Black Sea and Ancient Landscapes», in: J. CHAPMAN and P. DOLUKHANOV éds, Landscapes in flux: Central and Eastern Europe in Antiquity, p. 115-129.

SHNUKOV 1981

E. SHNUKOV, Geology of the Black Sea Shelf, Kiev.

STUIVER 1993

M. Stuiver, T.F. BRaZianas, « Modeling atmospheric $14 \mathrm{C}$ influences and radiocarbon ages of marine samples back to $10000 \mathrm{BC} »$, Radiocarbon 35/1, p. 137-189. 


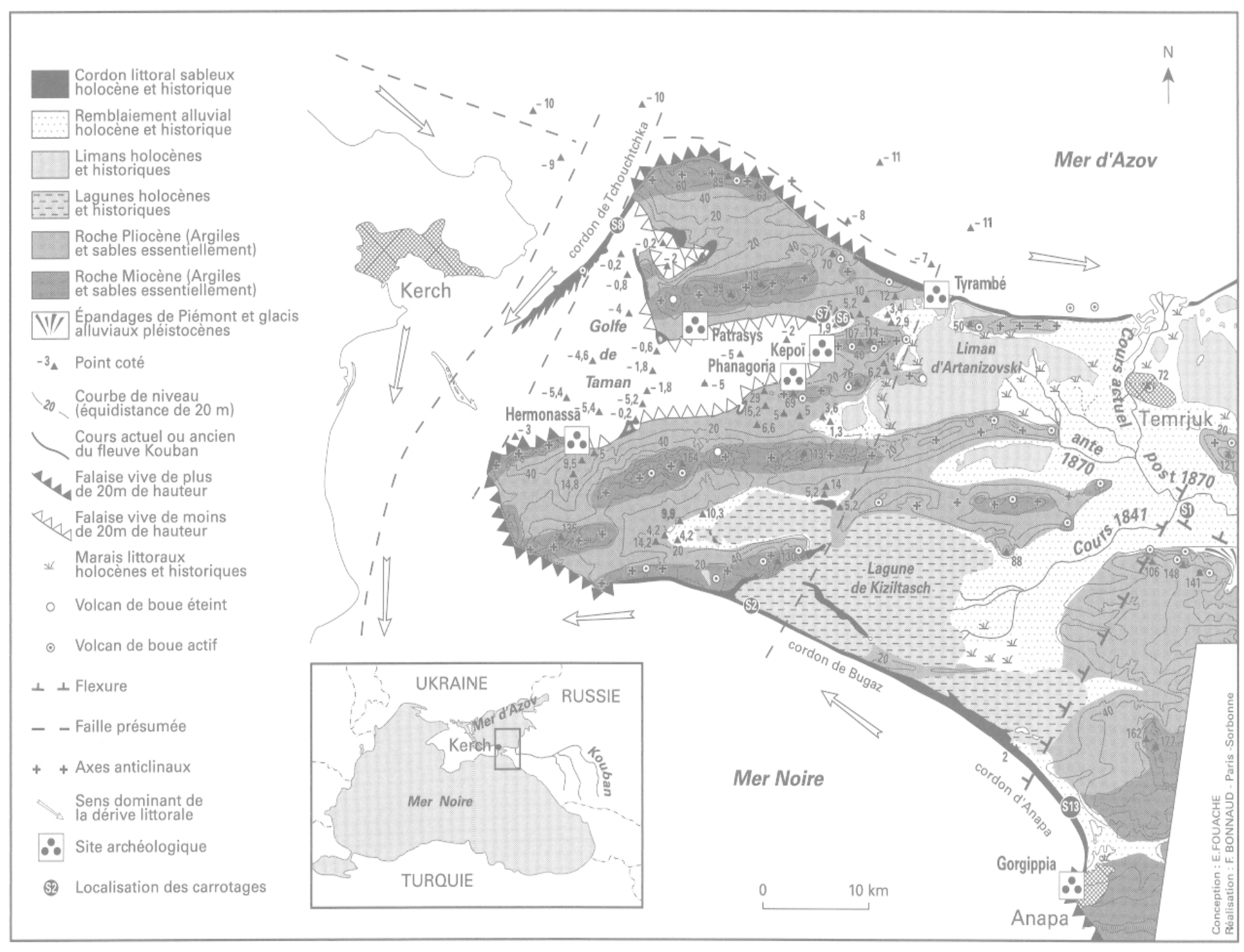

Fig. 1 - Carte géomorphologique de la presqu'île de Taman 


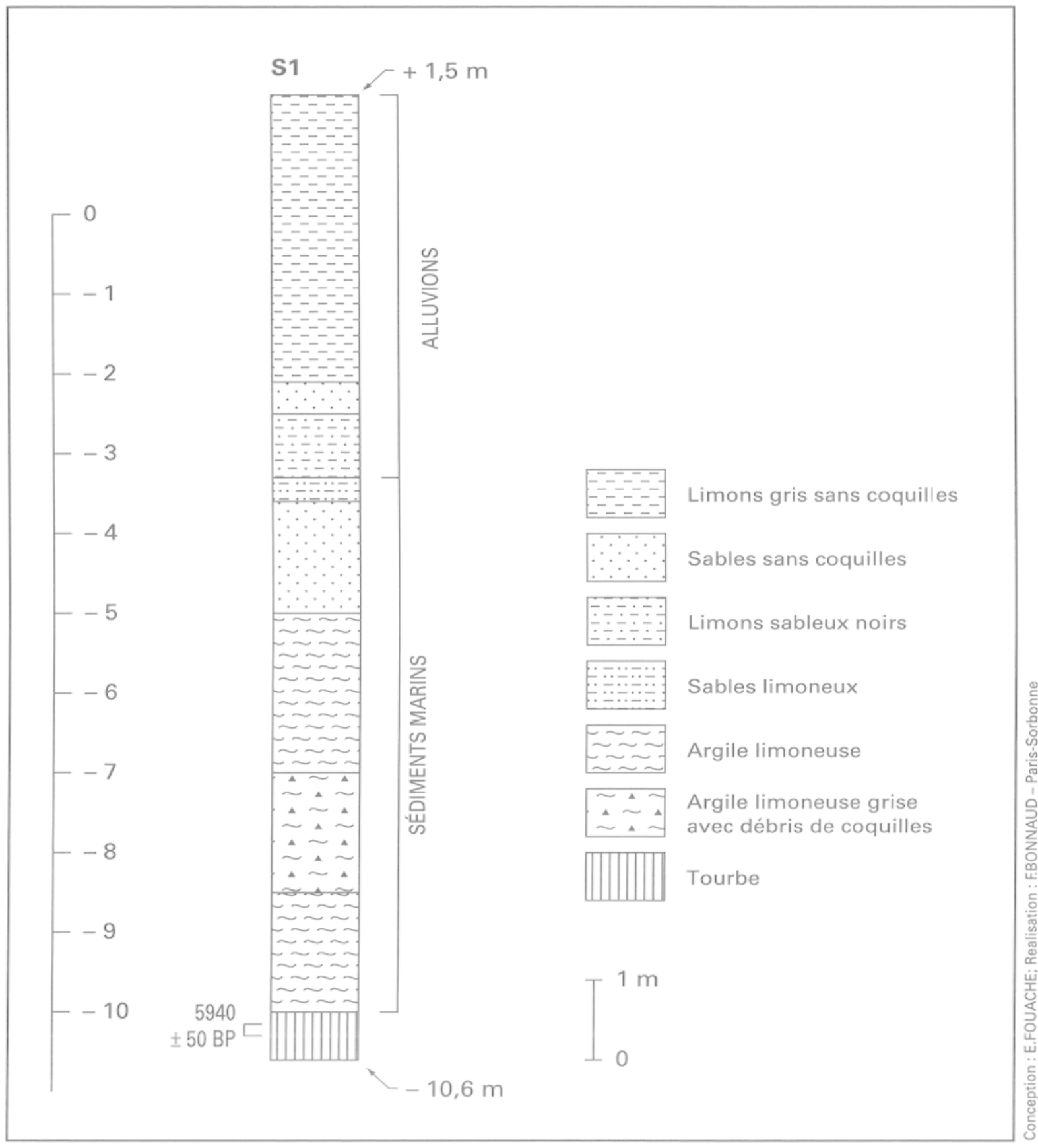

Fig. 2 - Profil sédimentologique simplifié du carrotage S 1 


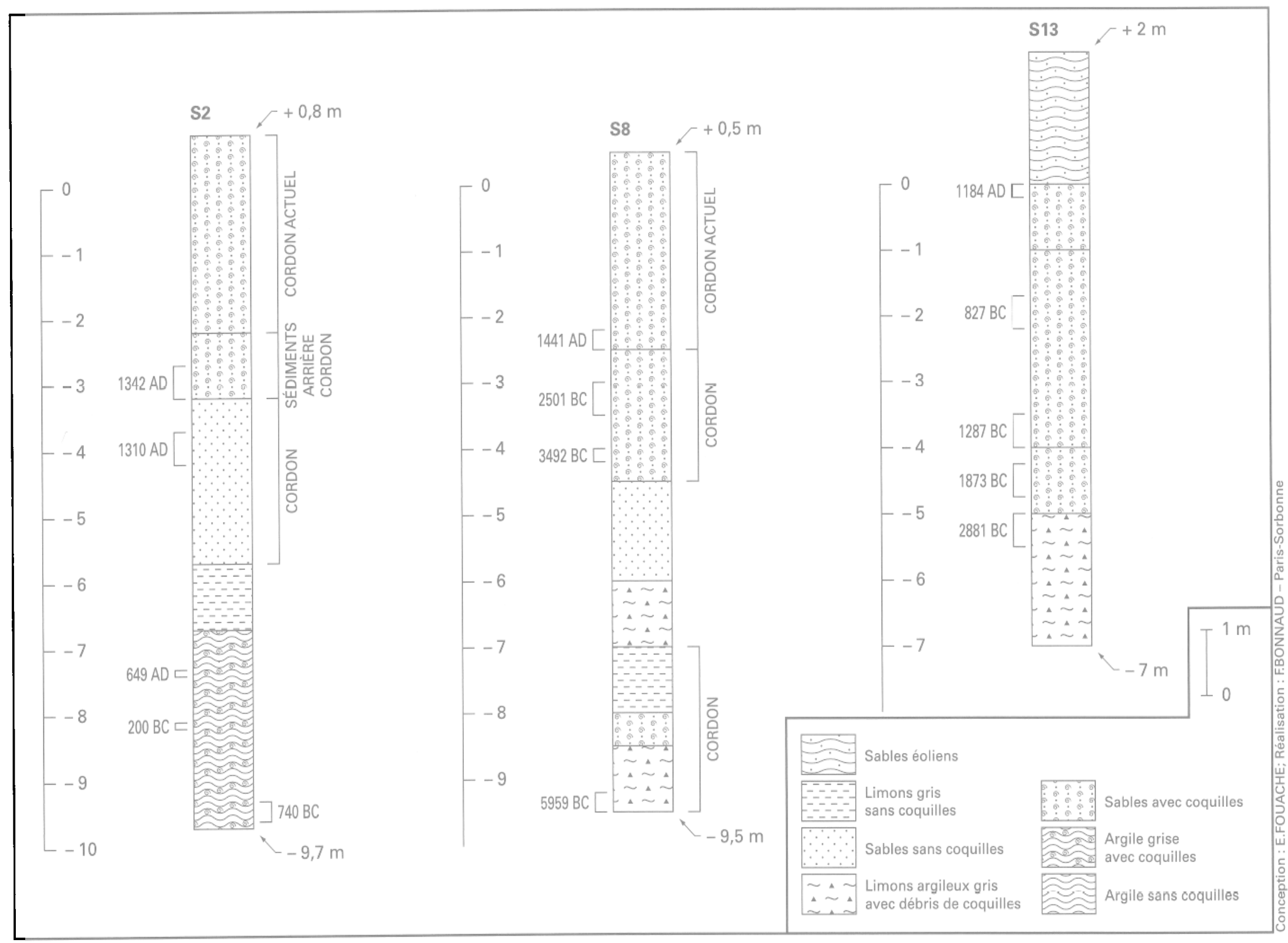




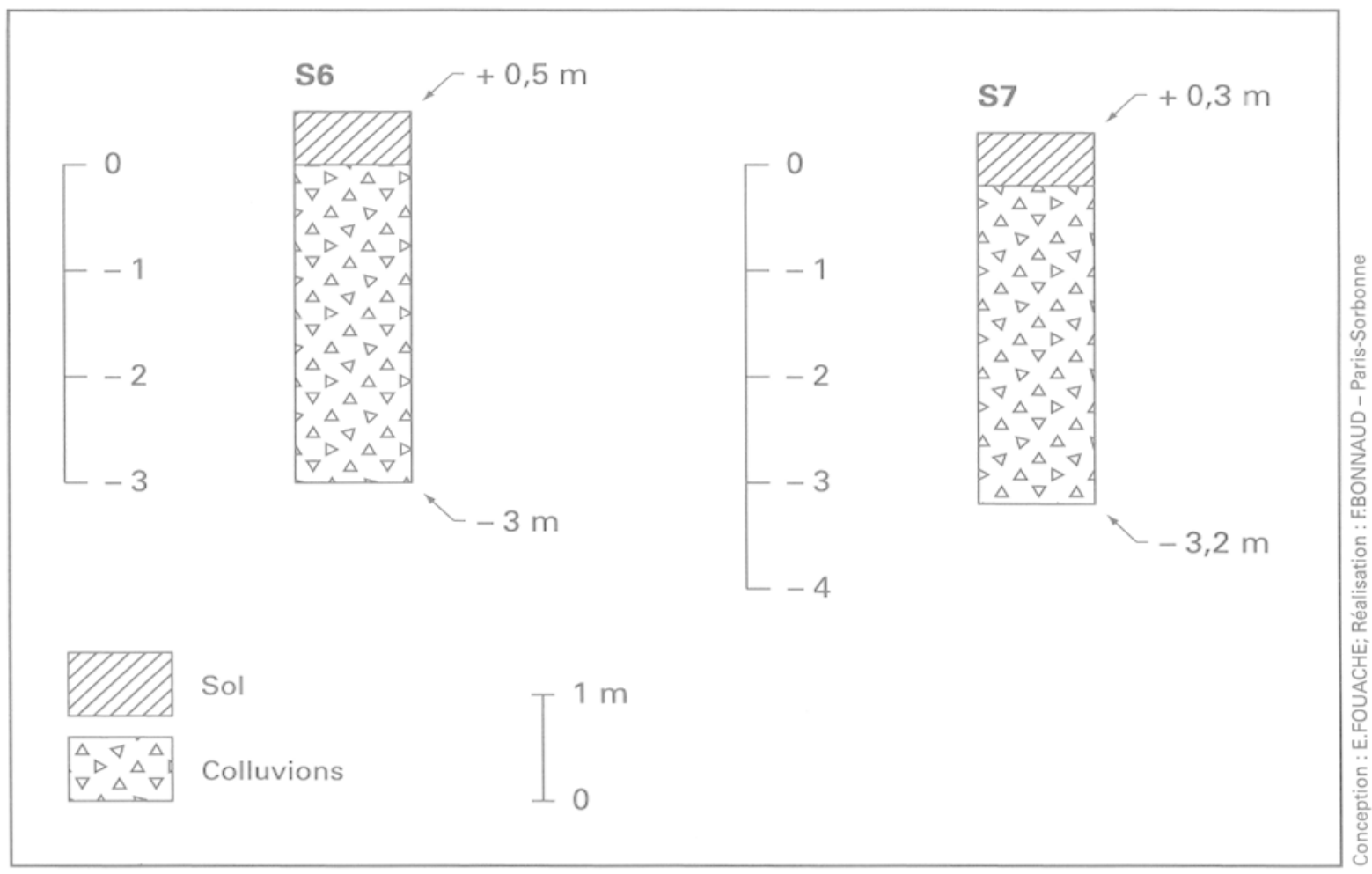

Fig. 4 - Profils sédimentologiques simplifiés des carrotages S6 et S7 

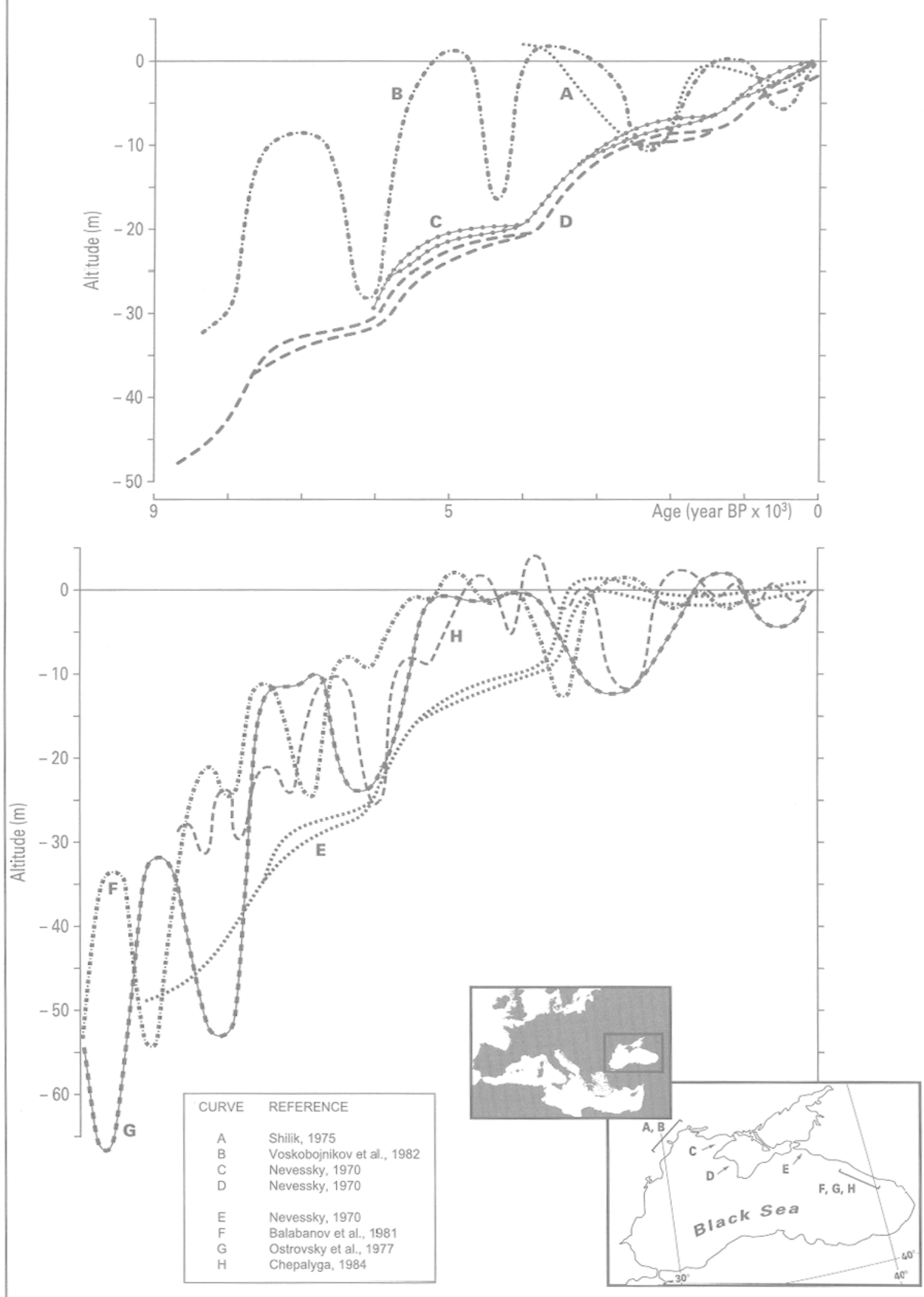

Fig. 5 - Reconstitutions des fluctuations holocènes du niveau marin dans la mer Noire proposées par différents auteurs (adapté de Pirazzoli, 1941) 